

\section{INTERACTION OF FAST NEUTRONS WITH ${ }^{4} \mathrm{He},{ }^{3} \mathrm{He}$, and ${ }^{1} \mathrm{H}$ :}

\section{ADDITIONAL AND IMPROVED DATA}

by

M. Drosg

\section{AESTRACT}

An improvement of previous elastic scattering data of neutrons in the $20-\mathrm{MeV}$ range was made possible by the improved knowledge of the detection efficiency of the neutron detector. Thus revised absolut differgntial cross-section data for ${ }^{3} \mathrm{He}(n, n){ }^{3} \mathrm{He}$ and $\mathrm{He}(n, n){ }^{4}$ He were obtained with smaller uncertainties. Cross sections of nonelastic $n-H \in$ reactions were obtained from recent data of the reciprocal reactions by detajled balance calculations.

In addition, incomplete angular distributions for the reaction $H(n, n) H$ between $22.5 \mathrm{MeV}$ and $28.5 \mathrm{MeV}$ are presented.

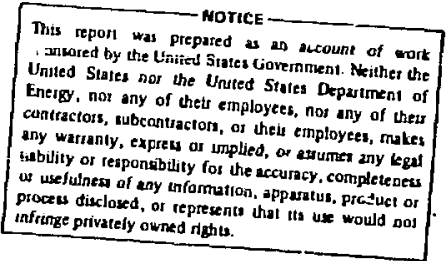




\section{INTRODUCTION}

For more than 10 years the same neutron detector (described in Rer. 1) has been used for measuring fast neutrons in few nucleon experiments (e.B., Refs. 2-4). As was shown in a recent paper ${ }^{5}$ the relative efficiency curve of this detector is now known with an uncertainty of less than $2 \%$ up to neutron energies of about $30 \mathrm{MeV}$. This compares with an uncertainty of 5 to $10 \%$ for the oldest efficiency curves used ${ }^{3}$ (see Fig. 1). If the pulse height bias has been set the same way during all these experiments and if the efficiency of the detector has not changed over these years, an improvement of the old data is possible just by using the new efficiency curve instead of the previous one.

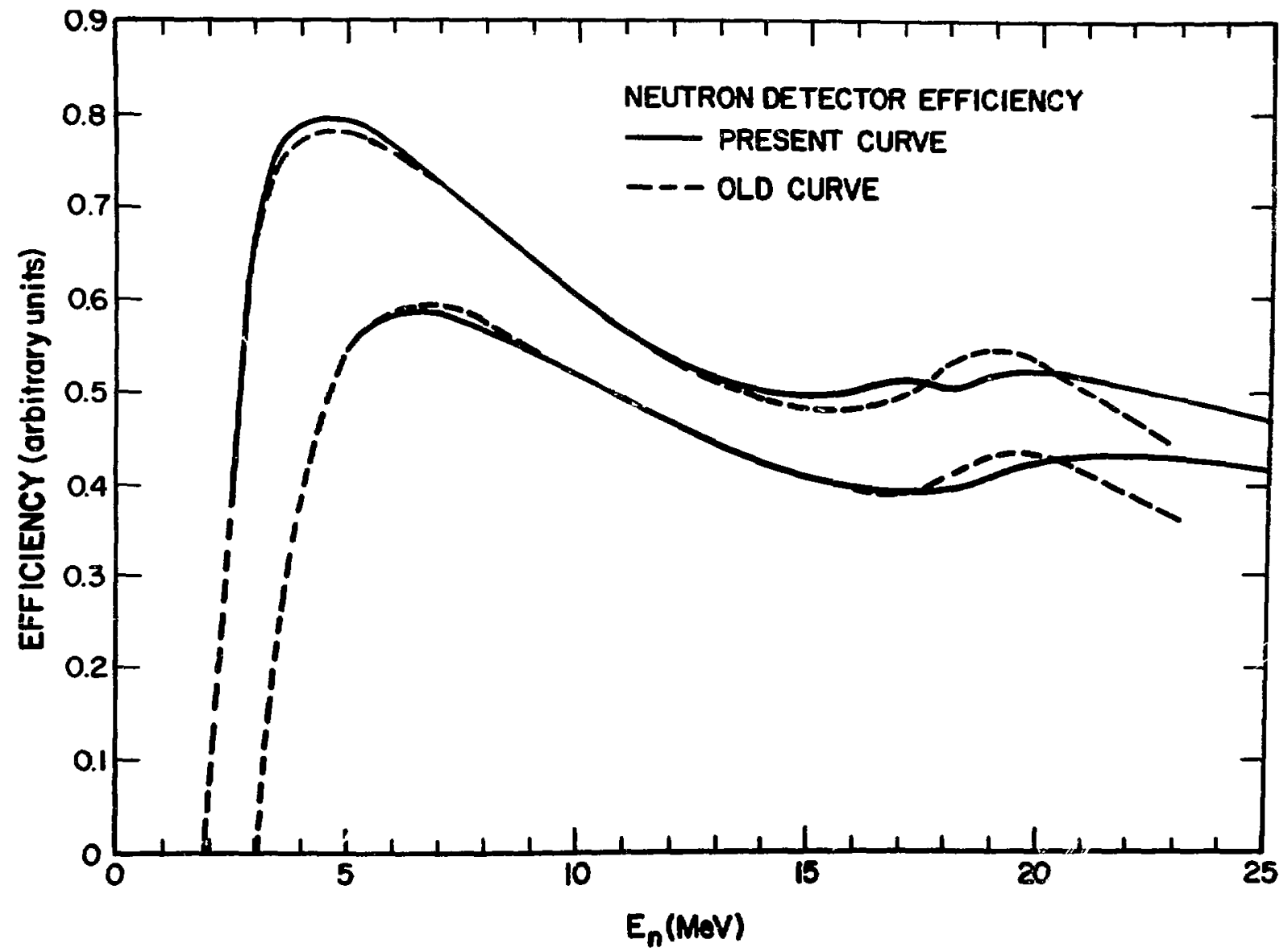

Fig. 1. Comparison of old efficiency curves (dashed, from Ref. 3) with latest ones (full, from Ref, 5). 
I1. PROCEDURE

In the course of data taking for the neutron production dat $\bar{z}^{5}$ from 1971 through 1975, no change in the neutron yjeld was observed when measuring the same angular distribution. This indicates that a change of the energy dependence of the neutron detection efficiency was negligible over these years for the biases used $(1 \times \mathrm{Cs}$ and $2 \times \mathrm{Cs})$. Therefore, it seems justified to assume that the shape of the efficiency curve did not change by more than $1 \%$ since 1969 in which year I joined the neutron time-of-flight group at the Los Alamos Scientific Laboratory (LASL). For data taken before this year a correction of the neutron detection efficiency would be less reliable anyway, since I have no personal knowledge how the pulse height bias was set.

Besides, I do not have raw data available as in the other cases. Therefore, I did not revise the ${ }^{2} \mathrm{H}(\mathrm{n}, \mathrm{n})^{2} \mathrm{H}$ and the ${ }^{3} \mathrm{H}(n, n)^{3} \mathrm{H}$ data, ${ }^{2}$ which were al so measured with the same detector.

Thus, only the data for the reaction ${ }^{4} \mathrm{He}(n, n)^{4} \mathrm{He},(\operatorname{Ref} . j)$ and ${ }^{3} \mathrm{He}(n, n){ }^{3} \mathrm{He}(\operatorname{Ref} .4)$ were revised.

The revision was done in 2 steps. First, the new efficiency was applied to the calibration runs determining the absolute scale. Then the new energy dependence of the efficiency curve was introduced. Since the shape of the angular distributions did not change drastically, the previous multiple scattering corrections were maintained.

In addition, integrated cross sections for the reactions ${ }^{4} \mathrm{He}(n, d)^{3} \mathrm{H}$, ${ }^{3} \mathrm{He}(n, p){ }^{3} \mathrm{H}$, and ${ }^{3} \mathrm{He}(n, d)^{2} \mathrm{H}$ were calculated from the corresponding timereversed reactions of Ref. 5 using the formulas given there.

Finally, relative angular distributions of the reaction ${ }^{1} H(n, n){ }^{1} H$ were obtained at $22.4 \mathrm{MeV}, 23.7 \mathrm{MeV}, 25.0 \mathrm{MeV}, 27.1 \mathrm{MeV}$, and $28.5 \mathrm{Mev}$ from the neutron yield of the original efficiency determination. 1 since the highenergy part of the final efficiency curve has been determined by means of neutron production cross sections, ${ }^{5}$ now these yields can be used to obtain (incomplete) angular distributions for $n-p$ scattering.

\section{RESULTS AND DISCUSSION}

The experimental conditions and the error contributions to the data are discussed in Refs. 1, 3: and 4. The uncertainties of the latest efficiency curves were discussed in Ref. 5 .

A. $n-{ }^{4}$ He data

Table I gives the revised absolute differential cross sections for the reaction ${ }^{4} \mathrm{He}(n, n)^{4} \mathrm{He}$ at $17.6,20.9$, and $23.7 \mathrm{MeV}{ }^{3}$ The changes which affect 
Absolute Differential Cross Sections for ${ }^{4} \mathrm{He}(n, n)^{4} \mathrm{He}$. Revised data from Ref. 3.

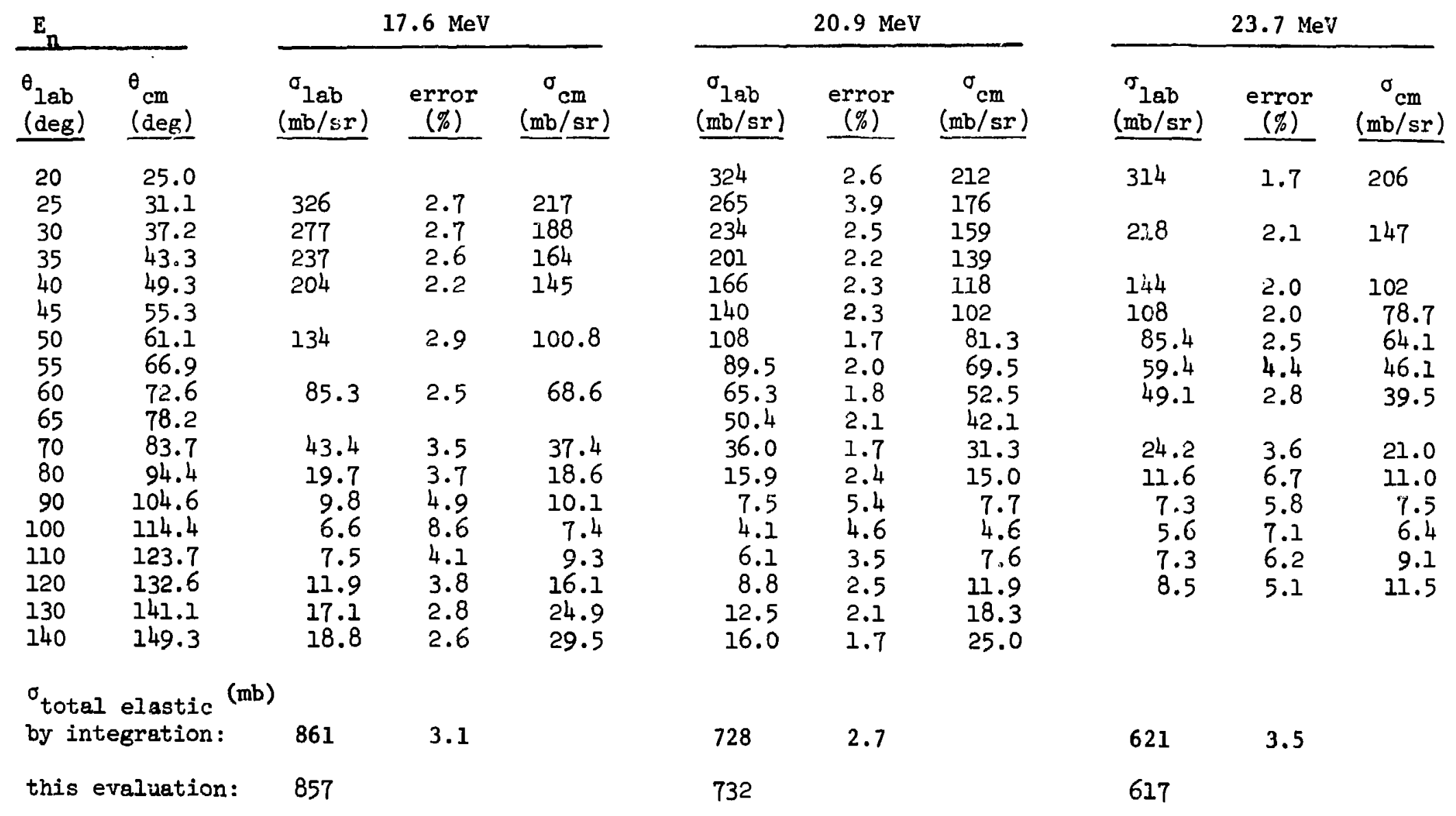


front angles most are within the error of 5 to $10 \%$ claimed for the previous efficiency curve. ${ }^{3}$ However, there is a marked improvement, especially for the 20.9-MeV data and the 23.7-MeV data since the changes remove a systematic error. In addition, the errors were revised, too, by taking into account the reduced uncertainty in the new efficiency curve.

Table IJ: gives the differential cross sections for the reaction ${ }^{4} \mathrm{He}(n, d){ }^{3} \mathrm{H}$ for neutron energies up to $36 \mathrm{MeV}$. The data are given in the center-of-mass system by their Legendre polynominal expansions in the form of

$$
\frac{d \sigma(E, \theta)}{d \Omega}=\frac{d \sigma\left(E, 0^{\circ}\right)}{d \Omega} \cdot \sum_{i} A_{i} P_{i}(\cos \theta)
$$

and were obtained by detailed balance conversion from data of the reciprocal reaction ${ }^{3} \mathrm{H}(\mathrm{d}, \mathrm{n})^{4} \mathrm{He}$. For neutron energies above $26 \mathrm{MeV}$, data of Ref. 5 were used; below that energy, those of Ref. 6 . Some smoothing in the transition range between these two data sets appeared to be necessary. Figure 2 summarizes the present knowledge of $\mathrm{n}-{ }^{4} \mathrm{He}$ cross sections. The curve up to 20 MeV was taken from ENDF/B-IV, which is based on the data of Goulding et al. 7 These data extend to about $30 \mathrm{MeV}$ as shown on the graph. It was found that the ENDF/B-IV data above $12 \mathrm{MeV}$ can be represented by the equation

$$
\sigma_{T}=\exp \left(-0.9181 \ln E_{n}+9.3860\right)
$$

with $E_{n}$ in MeV and $\sigma_{T}$ in mb, with deviations of less than $0.3 \%$. This equation is also in agreement with all the data up to $30 \mathrm{MeV}$, disregarding, however, the resonance above $22 \mathrm{MeV}$. (The data of Shamu et al. 8 must be downscaled by $1.5 \%$ to give best agreement with this expression.) Even an extrapolation to $150 \mathrm{MeV}$ seems justified, since the two high-energy data $\operatorname{sets}^{9,10}$ are in agreement with such an extrapolation. Subtracting the integrated cross section of the ${ }^{4} \mathrm{He}\left(n, d ;{ }^{3} \mathrm{H}\right.$ reaction from the total cross section gives the total elastic cross section up to $24.8 \mathrm{MeV}$, where a second nonelastic channel opens. As can be seen from the data in Tables I and II, the 3 elastic cross sections obtained in the present work by integration of the differential cross sections agree perfectly with these data. From the work of Tannenweld," who determined the relative contributions of the individual exit channels to the total cross section at $90 \mathrm{MeV}$, some idea about the energy dependence of the total elastic cross section and of that of the $d$ $+t$ channel can be obtained. 
${ }^{4} \mathrm{Ho}(\mathrm{n}, \mathrm{d})^{3} \mathrm{H}$. Recomended Values for $\sigma\left(0^{\circ} \mathrm{c} . \mathrm{m}.\right)$ Legendre coefficlents $\mathrm{h}_{1}$ and $\sigma($ total $)$

obtalned by Detalled Balance Conversion from ${ }^{3} \mathrm{H}(d, n)^{4}$ He. Coeffictents are multiplled by 1000.

\begin{tabular}{|c|c|c|c|c|c|c|c|c|c|c|c|c|c|c|c|c|c|c|}
\hline $\begin{array}{l}F_{n} \\
(m, v)\end{array}$ & $\begin{array}{l}\text { SIGMA-0 } \\
\text { (ab/sr) }\end{array}$ & $A_{0}$ & $A_{1}$ & $A_{2}$ & $A_{3}$ & $A_{4}$ & $A_{5}$ & $A_{6}$ & ${ }^{1} 7$ & ${ }^{A_{8}}$ & ${ }_{9}$ & $A_{10}$ & ${ }_{11}$ & ${ }_{i 2}$ & $A^{13}$ & $=a_{14}$ & $A_{15}$ & $\begin{array}{c}\text { SIFtA-T } \\
\text { (ab) }\end{array}$ \\
\hline 22.06 & 0.69 & 1000 & & & & & & & & & & & & & & & & 8.62 \\
\hline 22.08 & 3.22 & 1000 & & & & & & & & & & & & & & & & 40.4 \\
\hline 22.10 & 6.14 & 1000 & & & & & & & & & & & & & & & & 77.1 \\
\hline 22.12 & 7.36 & 1000 & & & & & & & & & & & & & & & & 92.5 \\
\hline 22.14 & 7.14 & 1000 & & & & & & & & & & & & & & & & 89.7 \\
\hline 22.16 & 6.49 & 1000 & & & & & & & & & & & & & & & & 81.5 \\
\hline 22.18 & 5.88 & 1000 & & & & & & & & & & & & & & & & 73.9 \\
\hline 22.20 & 5.35 & 1000 & & & & & & & & & & & & & & & & 67.2 \\
\hline 22.25 & 4.48 & 1000 & & & & & & & & & & & & & & & & 56.2 \\
\hline 22.30 & 4.00 & 1000 & & & & & & & & & & & & & & & & 50.3 \\
\hline 22.35 & 3.67 & 998 & 10 & -8 & & & & & & & & & & & & & & 46.1 \\
\hline 22.40 & 3.43 & 997 & 21 & -18 & & & & & & & & & & & & & & 43.0 \\
\hline 22.45 & 3.26 & 994 & 33 & -27 & & & & & & & & & & & & & & 40.7 \\
\hline 22.50 & 3.15 & 990 & 43 & -33 & & & & & & & & & & & & & & 39.2 \\
\hline 22.60 & 3.02 & 981 & 62 & -43 & & & & & & & & & & & & & & 37.2 \\
\hline 22.80 & 2.94 & 953 & 89 & -42 & & & & & & & & & & & & & & 35.2 \\
\hline 23.00 & 2.99 & 915 & 105 & -23 & 0 & 3 & & & & & & & & & & & & 34.4 \\
\hline 23.50 & 3.50 & 778 & 128 & 59 & $?$ & 12 & 12 & 4 & & & & & & & & & & 34.2 \\
\hline 24.00 & 4.42 & 640 & 133 & 141 & 24 & 20 & 32 & 10 & & & & & & & & & & 35.5 \\
\hline 24.50 & 5.99 & 523 & 128 & 206 & 45 & 28 & 51 & 18 & 1 & & & & & & & & & 39.4 \\
\hline 25.00 & C.21 & 431 & 121 & 253 & 63 & 36 & 67 & 26 & 3 & & & & & & & & & 414.5 \\
\hline 25.50 & 10.6 & 376 & 116 & 265 & 73 & 44 & 84 & 36 & 6 & & & & & & & & & 50.3 \\
\hline 26.00 & 13.0 & 341 & 116 & 250 & 73 & 56 & 103 & 47 & 10 & 3 & 1 & & & & & & & 55.6 \\
\hline 26.50 & 14.8 & 314 & 117 & 220 & 67 & 75 & 123 & 60 & 15 & 6 & 3 & & & & & & & 58.4 \\
\hline 27.00 & 16.1 & 292 & 110 & 192 & 53 & 97 & 148 & 74 & 21 & 9 & 4 & & & & & & & 59.1 \\
\hline 27.50 & 17.2 & 275 & 92 & 179 & 35 & 115 & 170 & 89 & 27 & 12 & 5 & 1 & & & & & & 59.4 \\
\hline 28.00 & 18.0 & 264 & 75 & 172 & 18 & 126 & 185 & 103 & 33 & 16 & 5 & 2 & 1 & & & & & 59.7 \\
\hline 29.00 & 19.1 & 247 & 64 & 160 & -10 & 132 & 192 & 136 & 40 & 23 & 7 & 4 & 2 & 3 & & & & 59.3 \\
\hline 30.00 & 20.4 & 231 & 61 & 149 & -27 & 126 & 193 & 169 & 45 & 30 & 8 & 7 & 2 & 5 & 1 & & & 59.1 \\
\hline 31.00 & 21.7 & 216 & 62 & 138 & -35 & 115 & 190 & 196 & 49 & 36 & 10 & 9 & 3 & 7 & 2 & 2 & & 58.9 \\
\hline 32.00 & 23.1 & 202 & 65 & 128 & -35 & 103 & 185 & 216 & 53 & 42 & 11 & 12 & 3 & 8 & 3 & 4 & & 58.8 \\
\hline 33.00 & 24.7 & 189 & 70 & 119 & -30 & 89 & 180 & 230 & 58 & 49 & 13 & 14 & 3 & 8 & 3 & 4 & 1 & 58.6 \\
\hline 34.00 & 26.4 & 176 & 75 & 111 & -18 & 75 & 174 & 239 & 62 & 57 & 15 & 16 & 3 & 8 & 1 & 3 & 3 & 58.4 \\
\hline 36.00 & 30.3 & 153 & is3 & 95 & 6 & 50 & 163 & 246 & 73 & 76 & 20 & 23 & 4 & 6 & -5 & 2 & 5 & 58.1 \\
\hline
\end{tabular}




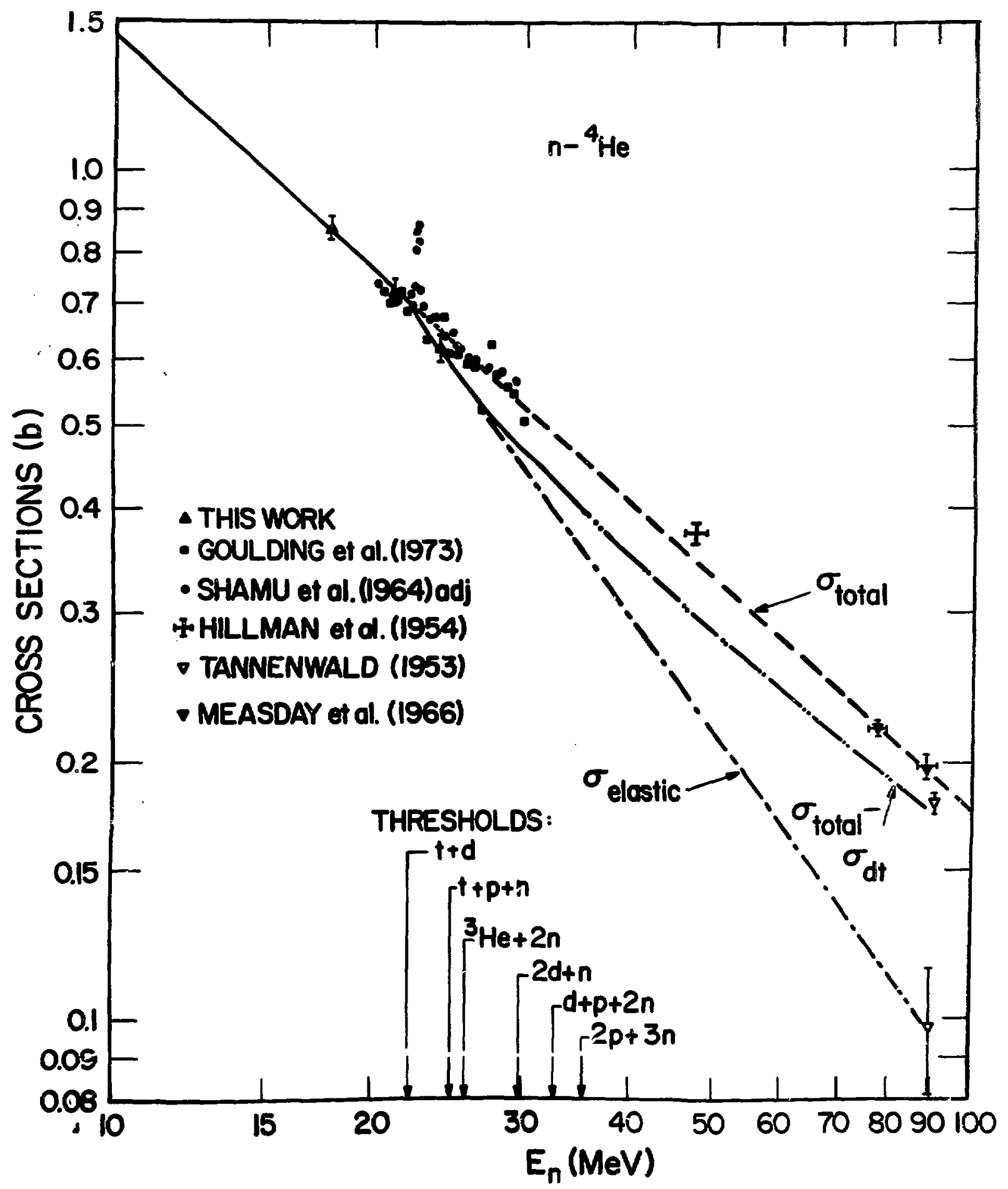

Fig. 2. Cross sections for the interactions of fast neutrons with ${ }^{4} \mathrm{He}$. Dashed-dotted curves are only meant to guide the eye. 
B. $n-{ }^{3}$ He data

Table III gives the revised absolute differential cross sections for the reaction ${ }^{3} \mathrm{He}(n, n){ }^{3} \mathrm{He}$ at $12.0,13.6,14.4$, and $23.7 \mathrm{MeV}{ }^{4}$ The data at $7.9 \mathrm{MeV}$ did not change. The data between 12 and $14.4 \mathrm{MeV}$ did not change much, actually the changes are within the uncertainty of the present efficiency curve. The $12 \mathrm{MeV}$ data include the results from measuring the gaseous sample as described in the original paper. The changes of the forward angle data of the $23.7 \mathrm{MeV}$ distribution are, however, not negligible due to the rather severe change of the efficiency curve at higher energies. Again, the shape error was revised as well, incorporating the uncertainty of the latest efficiency curve.

Table IV gives the differential cross sections for the reaction $3_{\mathrm{He}}(\mathrm{n}, \mathrm{p}){ }^{3} \mathrm{H}$ for neutron energies up to $19 \mathrm{MeV}$ in the same way as discussed above.

Table $V$ does the same for the reaction ${ }^{3} H e(n, d)^{2} H$ for neutron energies up to $21.2 \mathrm{MeV}$.

The uncertainties in the above representation of the nonelastic $n-H e$ interactions are discussed in Refs. 5 and 6 , respectively, where the unconverted numbers were taken from.

The energy dependence of the integral cross sections is show in Fig. 3 . The total cross section is due to Goulding et al. 7 Below $3 \mathrm{MeV}$ the old LASL $\operatorname{data}^{12}$ are also shown. The curve for elastic scattering was obtained by subtraction of $\sigma_{n, p}$ and $\sigma_{n, d}$ from the total cross section at lower energies and by integration of differential cross sections of the reaction ${ }^{3} \mathrm{He}(\mathrm{n}, \mathrm{n})^{3} \mathrm{He}{ }^{(\operatorname{Ref} .13)}$ and its charge-symmetric counterpart ${ }^{3} \mathrm{H}(\mathrm{p}, \mathrm{p})^{3} \mathrm{H}$ (see Ref. 4 for details). Below about $4 \mathrm{MeV}$ the curve for the elastic cross section looks somewhat strange. This is probably due to too low total cross sections near the resonance.

Subtracting all known partial cross sections from the total yields the cross section for 3- and 4-body breakup with thresholds at 7.3 and $10.3 \mathrm{MeV}$, respectively. This was done in Table VI and Fig. 4. Contrary to previous assumptions, this cross section is not negligible even at $10 \mathrm{MeV}$. This finding is supported by the charge symmetric breakup of tritium by protons which starts to be noticeable near the $2 n$ threshold as well. 5 The inset in Fig. 4 shows that the energy dependence of the zero degree differential cross section of p-t breakup is very similar to that of the total cross section of 
TABLE III

Absolute Differentlal Cross Sections for ${ }^{3} \mathrm{He}(n, n)^{3} \mathrm{He}$.

Revised Data from Ref. 4.

\begin{tabular}{|c|c|c|c|c|c|c|c|c|c|c|c|c|c|c|c|c|}
\hline \multirow{2}{*}{$\begin{array}{c}E_{n} \\
\cos \theta c \cdot m .\end{array}$} & \multicolumn{4}{|c|}{$12.00 \mathrm{MeV}$} & \multicolumn{4}{|c|}{$13.60 \mathrm{MeV}$} & \multicolumn{4}{|c|}{$14.40 \mathrm{MeV}$} & \multicolumn{4}{|c|}{$23.70 \mathrm{MeV}$} \\
\hline & $\begin{array}{l}\theta_{1 a b} \\
(d a g) \\
\end{array}$ & $\begin{array}{c}\sigma_{l a b} \\
(\mathrm{ab} / \mathrm{ar})\end{array}$ & $\begin{array}{c}\text { error } \\
1 \\
\end{array}$ & $\begin{array}{c}\sigma_{c . m} \\
(\mathrm{ob} / \mathrm{sr}) \\
\end{array}$ & $\begin{array}{l}\theta_{1 \mathrm{ab}} \\
(\mathrm{deg}) \\
\end{array}$ & $\begin{array}{c}\sigma_{1 \mathrm{ab}} \\
(\mathrm{mb} / \mathrm{sr})\end{array}$ & $\begin{array}{l}\text { error } \\
1 \\
\end{array}$ & $\begin{array}{c}\sigma_{c}, \pi \\
(m b / g r)\end{array}$ & $\begin{array}{l}\theta_{1 \mathrm{ab}} \\
\text { (dag) } \\
\end{array}$ & $\begin{array}{c}\sigma_{1 \mathrm{ab}} \\
(\mathrm{mb} / \mathrm{sr})\end{array}$ & $\begin{array}{c}\text { error } \\
8 \\
\end{array}$ & $\begin{array}{c}\sigma_{c \cdot m} . \\
(\mathrm{mb} / \mathrm{sr})\end{array}$ & $\begin{array}{l}\theta_{1 \mathrm{ab}} \\
\text { (deg) }\end{array}$ & $\begin{array}{c}\sigma_{1 a b} \\
(a b / s r)\end{array}$ & $\begin{array}{c}\text { error } \\
5 \\
\end{array}$ & $\begin{array}{c}\sigma_{\mathrm{c}} \cdot \mathrm{m} . \\
(\mathrm{mb} / \mathrm{s} r)\end{array}$ \\
\hline 0.90 & 19.5 & 532 & 3.3 & 307 & 19.4 & 464 & 3.7 & 267 & 19.3 & 430 & 3.3 & 248 & 19.4 & 283 & 3.9 & 162 \\
\hline 0.80 & 28.0 & 412 & 4.4 & 246 & 27.9 & 340 & 3.8 & 203 & 27.7 & 336 & 3.1 & 200 & 27.8 & 205 & 3.4 & 122 \\
\hline 0.70 & 34.6 & 297 & 1.8 & 184 & 34.6 & 269 & 3.4 & 166 & 34.5 & 261 & 3.3 & 162 & 34.6 & 146 & 4.0 & 90.3 \\
\hline 0.60 & 40.5 & 230 & 2.2 & 147 & 40.5 & 206 & 3.2 & 133 & 40.5 & 187 & 3.5 & 120 & 40.5 & 101 & 5.4 & 64.7 \\
\hline 0.55 & 43.4 & 196 & 1.8 & 128 & 43.4 & 171 & 2.5 & 112 & 43.4 & 164 & 3.2 & 108 & 43.4 & 79.6 & 4.8 & 52.1 \\
\hline 0.40 & 51.3 & 120 & 6.4 & 84.0 & 51.4 & 105 & 3.9 & 73.2 & 51.4 & 103 & 3.6 & 72.0 & 51.3 & 50.6 & 6.8 & 35.3 \\
\hline 0.25 & 58.9 & 75.0 & 6.2 & 56.1 & 58.8 & 68.7 & 4.1 & 51.3 & 58.8 & 65.9 & 3.9 & 49.2 & 58.9 & 28.5 & 6.2 & 21.3 \\
\hline 0.10 & 66.4 & 44.7 & 5.6 & 36.1 & 66.5 & 36.7 & 4.8 & 29.7 & 66.3 & 32.8 & 5.6 & 26.5 & 66.3 & 17.5 & 8.5 & 14.0 \\
\hline 0.00 & 71.6 & 27.8 & 4.1 & 23.7 & 71.6 & 24.5 & 4.4 & 20.9 & 71.4 & 23.6 & 4.0 & 20.1 & 71.5 & 10.5 & 18.0 & 8.9 \\
\hline-0.10 & 76.8 & 17.6 & 7.5 & 15.9 & 76.8 & 17.0 & 5.9 & 15.4 & 76.6 & 14.3 & 5.5 & 12.9 & 76.6 & 7.4 & 17.6 & 6.7 \\
\hline-0.30 & 87.8 & 7.6 & 5.2 & 7.8 & 87.8 & 6.2 & 5.0 & 6.5 & 87.8 & 6.1 & 5.7 & 6.3 & 87.8 & 5.5 & 13.1 & 5.7 \\
\hline-0.50 & 100.9 & 7.4 & 7.1 & 9.0 & 100.8 & 6.0 & 5.9 & 7.3 & 100.7 & 5.1 & 6.5 & 6.2 & 100.7 & 4.1 & 17.9 & 5.1 \\
\hline-0.60 & 108.4 & 11.5 & 5.8 & 15.4 & 108.4 & 8.6 & 5.1 & 11.5 & 108.5 & 7.9 & 4.6 & 10.6 & 108.3 & 4.4 & 12.6 & 5.9 \\
\hline-0.715 & 118.2 & 16.8 & 5.8 & 25.4 & 118.5 & 12.5 & 5.5 & 18.9 & 118.5 & 12.4 & 3.7 & 18.7 & 118.5 & $5 . j$ & 10.6 & 8.4 \\
\hline \multicolumn{2}{|c|}{$\begin{array}{l}\text { Scal e errors } \\
\text { (not :naluded) }\end{array}$} & & 2.3 & & & & 3.1 & & & & 2.8 & & & & 2.9 & \\
\hline $\begin{array}{l}\text { toal }= \\
(\pi b)\end{array}$ & $7: 3^{*}$ is & 1029 & 3.6 & & & 902 & 3.8 & & & 850 & 3.8 & & & 489 & 3.9 & \\
\hline
\end{tabular}


TABLE IV

${ }^{3}$ He $(n, p)^{3}$. Recommended Values for $\sigma\left(0^{\circ} \varepsilon\right.$, m. $)$, Legendre Coefficients $A_{1}$ and "(total).

obtained by Detafled Balance Conversion from ${ }^{3} \mathrm{H}(\mathrm{p}, \mathrm{n})^{3}$ He. Coefficients are multiplied by 1000 .

\begin{tabular}{|c|c|c|c|c|c|c|c|c|c|c|c|c|}
\hline $\begin{array}{c}E_{n} \\
(M e V)\end{array}$ & $\begin{array}{l}\text { SIGMA-0 } \\
(\text { (ab/sr) }\end{array}$ & $A_{0}$ & $A_{1}$ & $\underline{A_{2}}$ & $\mathrm{~A}_{3}$ & $A_{4}$ & $A_{5}$ & ${ }_{6}^{A_{6}}$ & $A_{7}$ & $A_{8}$ & ${ }_{9}$ & $\begin{array}{l}\text { SIGMA-T } \\
\text { (mb) }\end{array}$ \\
\hline 0.50 & 63.8 & 1163 & -450 & 287 & & & & & & & & 932 \\
\hline 0.60 & 61.0 & 1171 & -528 & 357 & & & & & & & & 897 \\
\hline 0.70 & 59.7 & 1174 & -589 & 415 & & & & & & & & 881 \\
\hline 0.80 & 59.4 & 1171 & -633 & 462 & & & & & & & & 875 \\
\hline 0.90 & 59.9 & 1162 & -663 & 500 & & & & & & & & 875 \\
\hline 1.00 & 61.6 & 1138 & -671 & 534 & & & & & & & & 880 \\
\hline 1.20 & 68.4 & 1036 & -623 & 595 & -8 & & & & & & & 889 \\
\hline 1.40 & 78.5 & 898 & -526 & 644 & -16 & & & & & & & 886 \\
\hline 1.60 & 86.8 & 804 & -473 & 693 & -24 & & & & & & & 877 \\
\hline 1.80 & 90.3 & 761 & -454 & 727 & -34 & & & & & & & 864 \\
\hline 2.00 & 89.7 & 744 & -450 & 754 & -48 & & & & & & & 839 \\
\hline 2.50 & 81.2 & 738 & -473 & 835 & -100 & & & & & & & 753 \\
\hline 3.00 & 66.6 & 769 & -530 & 915 & -176 & 26 & -4 & & & & & 643 \\
\hline 3.50 & 54.1 & 809 & -589 & 996 & -269 & 64 & -13 & 2 & & & & 550 \\
\hline 4.00 & 44.4 & 854 & -647 & 1077 & -380 & 114 & -22 & 9 & -5 & & & 476 \\
\hline 4.50 & 36.9 & 910 & -711 & 1154 & -504 & 176 & -33 & 16 & -8 & & & 422 \\
\hline 5.00 & 30.4 & 974 & -773 & 1226 & -642 & 247 & -44 & 22 & -11 & & & 373 \\
\hline 5.50 & 25.5 & 1046 & -833 & 1285 & -778 & 323 & -58 & 28 & -13 & & & 335 \\
\hline 6.00 & 21.7 & 1119 & -883 & 1323 & -905 & 401 & -73 & 34 & -16 & & & 305 \\
\hline 6.50 & 19.2 & 1165 & -900 & 1320 & -999 & 479 & -87 & 39 & -17 & & & 281 \\
\hline 7.00 & 17.5 & 1185 & -885 & 1279 & -1052 & 546 & -100 & 45 & -18 & & & 261 \\
\hline 7.50 & 16.5 & 1179 & -842 & 1205 & -1064 & 601 & -110 & 49 & -18 & & & 244 \\
\hline 8.00 & 16.0 & 1148 & -771 & 1100 & -1030 & 634 & -117 & 53 & -17 & & & 231 \\
\hline 8.50 & 16.1 & 1090 & -677 & 968 & -950 & 645 & -117 & 55 & -14 & & & 221 \\
\hline 9.00 & 16.6 & 1020 & -575 & 835 & -852 & 640 & -114 & 56 & -10 & & & 213 \\
\hline 10.00 & 17.9 & 867 & -399 & 630 & -659 & 614 & -105 & 56 & -4 & & & 195 \\
\hline 11.00 & 19.6 & 726 & -259 & 493 & -487 & 564 & -93 & 55 & 1 & & & 179 \\
\hline 12.00 & 21.4 & 612 & -151 & 404 & -351 & 508 & -81 & 53 & 5 & 1 & & 165 \\
\hline 13.00 & 23.2 & 521 & -67 & 343 & -246 & 456 & -68 & 51 & 8 & 4 & -2 & 152 \\
\hline 14.00 & 24.7 & 452 & -3 & 302 & -171 & 411 & -54 & 50 & 11 & 7 & -5 & 140 \\
\hline 15.00 & 36.0 & 398 & 45 & 276 & -115 & 370 & -42 & 51 & 13 & 10 & -6 & 130 \\
\hline 17.00 & 37.8 & 323 & 110 & 247 & -34 & 311 & -24 & 52 & 15 & 11. & -11 & 113 \\
\hline 19.00 & 29.1 & 270 & 151 & 230 & 24 & 263 & -7 & $5 \mathrm{~N}$ & 16 & 13 & -14 & 99 \\
\hline
\end{tabular}


TABLE $V$

${ }^{3} \mathrm{He}(n, d)^{2} \mathrm{H}$. Recommended values for $\sigma\left(0^{\circ} \mathrm{cm} . \mathrm{m}\right)$, Legendre Coefficients $A_{1}$ and

$\sigma$ (total). Obtained by Detailed Balance Conversion from ${ }^{2} H(d, n)^{3}$ He. Coefficients are multiplied by 1000.

\begin{tabular}{|c|c|c|c|c|c|c|c|c|c|c|c|}
\hline $\begin{array}{c}\begin{array}{c}E_{n} \\
(M e V)\end{array} \\
\end{array}$ & $\begin{array}{l}\text { SIGMA-0 } \\
\text { (mb/sr) }\end{array}$ & $A_{0}$ & $A_{2}$ & $\mathrm{~A}_{4}$ & $A_{6}$ & ${ }_{8}$ & $A_{10}$ & $\mathrm{~A}_{12}$ & $\mathrm{~A}_{14}$ & $\mathrm{~A}_{16}$ & $\begin{array}{c}\text { SIGMA -T } \\
(\mathrm{mb})\end{array}$ \\
\hline $4 \cdot>0$ & 0.22 & 602 & 381 & 17 & & & & & & & 1.64 \\
\hline 4.60 & 0.65 & 545 & 419 & 36 & & & & & & & 4.45 \\
\hline 4.70 & 1.24 & 505 & 437 & 58 & & & & & & & 7.84 \\
\hline 4.80 & 1.96 & 473 & 444 & 83 & & & & & & & 11.7 \\
\hline 4.90 & 2.72 & 446 & 445 & 109 & & & & & & & 15.3 \\
\hline 5.00 & 3.52 & 424 & 441 & 135 & & & & & & & 18.6 \\
\hline 5.20 & 5.18 & 386 & 425 & 182 & 7 & & & & & & 25.2 \\
\hline 5.40 & 6.83 & 355 & 407 & 222 & 16 & & & & & & 30.5 \\
\hline 5.60 & 8.45 & 330 & 389 & 256 & 25 & & & & & & 35.0 \\
\hline 5.80 & 10.1 & 307 & 375 & 283 & 35 & & & & & & 39.0 \\
\hline 6.00 & 11.7 & 290 & 359 & 305 & 46 & & & & & & 42.7 \\
\hline 6.50 & 15.8 & 253 & 331 & 346 & 70 & & & & & & 50.3 \\
\hline 7.00 & 19.9 & 225 & 307 & 359 & 94 & 5 & & & & & 56.3 \\
\hline 7.50 & 23.6 & 206 & 289 & 377 & 114 & 14 & & & & & 61.1 \\
\hline$Q .00$ & 27.2 & 190 & 273 & 380 & 133 & 23 & 1 & & & & 64.8 \\
\hline 8.50 & 30.5 & 177 & 258 & 376 & 151 & 31 & 6 & 1 & & & 67.8 \\
\hline 9.00 & 33.5 & 167 & 247 & 370 & 165 & 39 & 9 & 2 & 1 & & 70.1 \\
\hline 9.50 & 36.1 & 158 & 240 & 362 & 178 & 47 & 11 & 2 & 2 & & 71.8 \\
\hline 10.00 & 38.3 & 152 & 233 & 354 & 190 & 53 & 13 & 3 & 2 & & 73.1 \\
\hline 11.00 & 41.6 & 143 & 223 & $\$ 339$ & 208 & 63 & 16 & 4 & 4 & & 74.0 \\
\hline 12.00 & 43.7 & 137 & 216 & 322 & 223 & 71 & 20 & 6 & 5 & & 75.1 \\
\hline 13.00 & 45.1 & 132 & 210 & 306 & 236 & 79 & 23 & 7 & 6 & 1 & 74.8 \\
\hline 14.00 & 45.8 & 128 & 206 & 290 & 245 & 87 & 27 & 8 & 6 & 3 & 73.8 \\
\hline 15.00 & 46.2 & 125 & 202 & 275 & 253 & 94 & 31 & 9 & 7 & 4 & 72.6 \\
\hline 16.00 & 46.2 & 122 & 198 & 261 & 260 & 101 & 34 & 11 & 8 & 5 & 71.0 \\
\hline 18.00 & 45.8 & 117 & 191 & 238 & 272 & 113 & 41 & 13 & 9 & 6 & 67.3 \\
\hline 21.20 & 44.6 & 108 & 183 & 215 & 280 & 129 & 53 & 17 & 10 & 5 & 60.6 \\
\hline
\end{tabular}




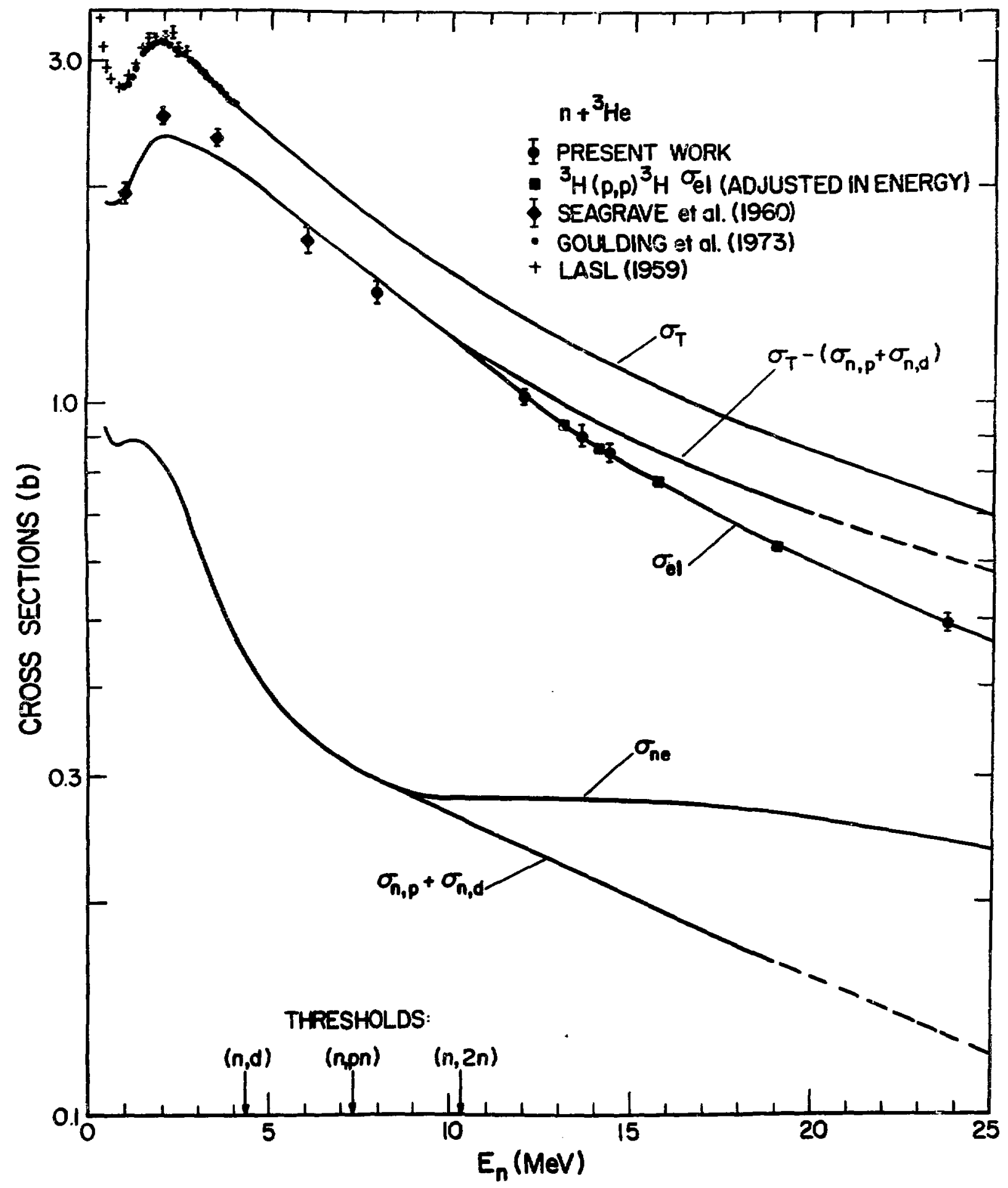

Fig. 3. Cross sections for interactions of fast neutrons with ${ }^{3} \mathrm{He}$. Dashed curves are not so well established as full curves. 
TABLE V:

$n-{ }^{3} \mathrm{He}$ Integral Cross Sections (in barns;

\begin{tabular}{|c|c|c|c|c|c|c|}
\hline$E_{n}$ & $\sigma_{\mathrm{T}}^{\mathrm{a}}$ & $\sigma_{n, p}$ & $\sigma_{n, d}$ & $\sigma_{\text {el }}$ & ${ }^{\sigma} \mathrm{n}, \mathrm{pn}$ & $\sigma_{n, 2 n}$ \\
\hline & & & & & Indivia & Irom curve \\
\hline 7.9 & $\begin{array}{r}1.792 \\
+0.018\end{array}$ & $\begin{array}{r}0.234 \\
+0.005\end{array}$ & $\begin{array}{r}0.064 \\
+0.001\end{array}$ & $\begin{array}{r}1.43 \\
+0.05\end{array}$ & $\begin{array}{r}0.06 \\
+0.05\end{array}$ & 0 \\
\hline 12.0 & $\begin{array}{r}1.315 \\
+0.013\end{array}$ & $\begin{array}{r}0.165 \\
+0.003\end{array}$ & $\begin{array}{r}0.075 \\
+0.001\end{array}$ & $\begin{array}{r}1.023 \\
+0.025\end{array}$ & $\begin{array}{r}0.05 \\
+0.03\end{array}$ & 0.04 \\
\hline 13.6 & $\begin{array}{r}1.185 \\
+0.012\end{array}$ & $\begin{array}{r}0.145 \\
+0.003\end{array}$ & $\begin{array}{r}0.074 \\
+0.001\end{array}$ & $\begin{array}{r}0.902 \\
\pm 0.029\end{array}$ & $\begin{array}{r}0.06 \\
+0.03\end{array}$ & 0.06 \\
\hline 14.4 & $\begin{array}{r}1.127 \\
+0.011\end{array}$ & $\begin{array}{r}0.136 \\
+0.003\end{array}$ & $\begin{array}{r}0.073 \\
+0.001\end{array}$ & $\begin{array}{r}0.856 \\
+0.024\end{array}$ & $\begin{array}{r}0.06 \\
+0.03\end{array}$ & 0.07 \\
\hline 23.7 & $\begin{array}{r}0.733 \\
+0.020\end{array}$ & $\begin{array}{r}0.074 \\
+0.005\end{array}$ & $\begin{array}{r}0.055 \\
+0.001\end{array}$ & $\begin{array}{r}0.492 \\
+0.014\end{array}$ & $\begin{array}{r}0.11 \\
+0.02\end{array}$ & - \\
\hline
\end{tabular}

a From Ref. 7 .

\section{C. n-D data}

Table VII gives relative differential cross sections for the reaction $1_{H(n, n)}{ }^{1} \mathrm{H}$. These data were originally taken to provide the efficiency curve at higher neutron energies (see Ref. 1). Later on, however, this part of the efficiency curve was determined by means of neutron production cross sections (see Ref. 5). Therefore, these measurements provide now experimental data for $n-p$ scattering. Although the errors, especially at the higher energies, are near $4 \%$ it seems wortbwhile to provide these data, since they match other $n-p$ data very closely in energy. Data of other authors are available at $22.5 \mathrm{MeV}^{14} 24.0 \mathrm{MeV},{ }^{15}, 16,1727.2 \mathrm{MeV},{ }^{15} 27.3 \mathrm{MeV},{ }^{18}$ and $28.4 \mathrm{MeV} .^{19}$ Except for 2 data points at $24 \mathrm{MeV}^{17}$ all these data are relative data like the present. By adjusting the data of corresponding energies by means of a Legendre fit to the well-known total cross section, 20 an absolute scale could be established. The adjusted data, together with their Legendre fits, are shown in Fig. 5. Although all of these data are in agreement with the YALE predictions for these cross sections, 20 they do not rule out a solution with lower $180^{\circ}$ values as shown in Fig. 5. Lower $180^{\circ}$ cross sections would explain discrepancies in counter telescope measurements for neutron energies around $25 \mathrm{MeV} .5,21$ 
$r_{1}-{ }^{3}$ He breakup. Actually, the curve obtained for $n-{ }^{3}$ he was overlaid on the p-I data points after adjusting the energy to have the threshold energies coinciding.
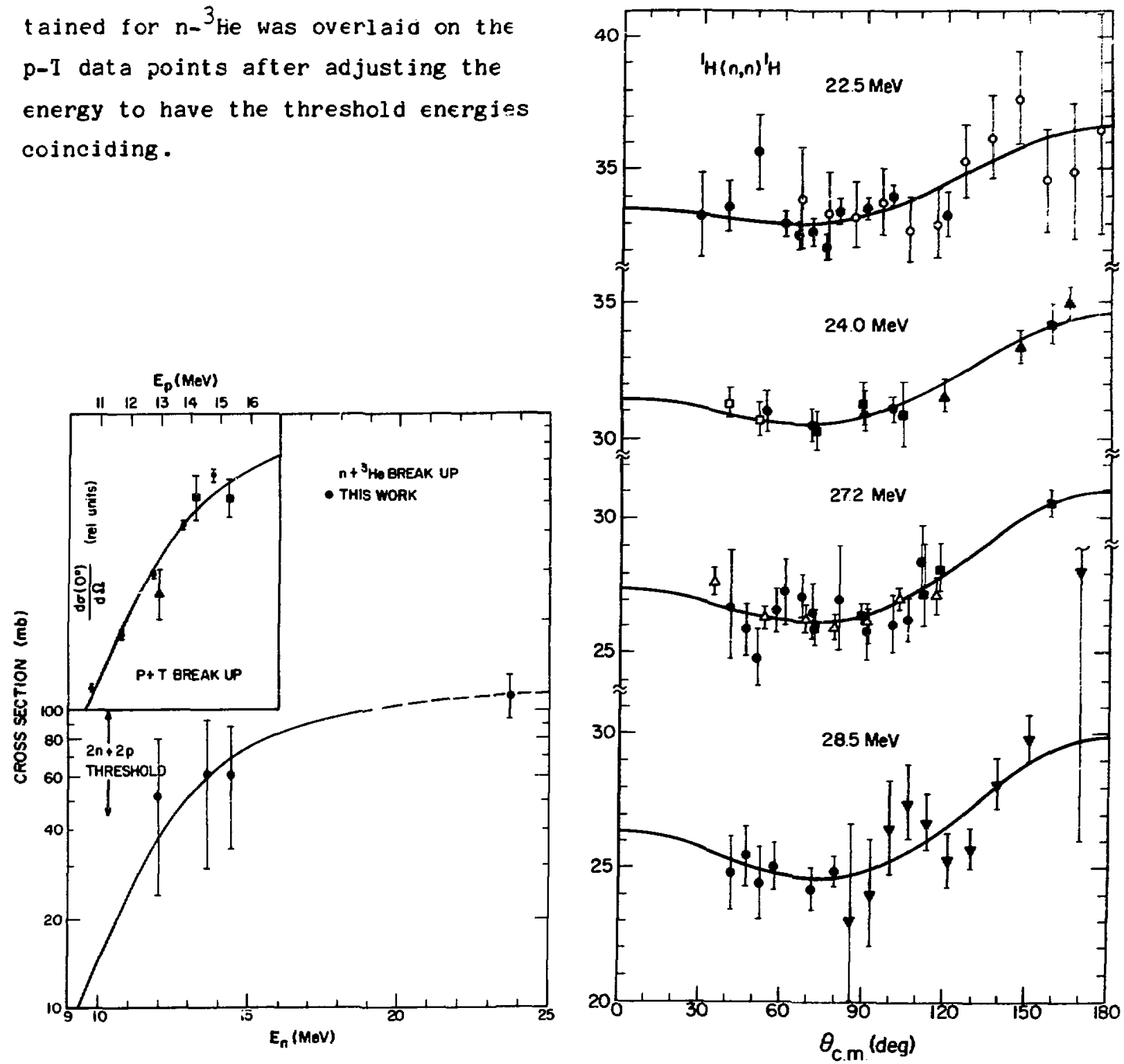

Fig. 4. Cross sections for the 3 - and 4-body breakup of $n-{ }^{3} \mathrm{He}$. The insert was taken from kef. 5 . It shows the same increase with energy for the chargesymmetric data.

F'ig. 5. Center-of-mass, differential cross-section data for $\mathrm{H}(n, n){ }_{\mathrm{H}}$ bet ween 22.5 and $26.5 \mathrm{Mev}$, given in $\mathrm{mb} / \mathrm{sr}$. Relative data were adjusted in scale so that the fit gave the correct total cross sections. All fits were forced to give a $196 \in$ er $180^{\circ}$ value than predicted by Yale. ${ }^{2}$ Full circles: present data; open circles: kief. 14; ful I squares: $k \in f .15$; full triangles at 24 $\mathrm{MeV}$, Ref. 16; open squares: $\mathrm{k} \in \mathrm{f} .17$; open triangles: Ref. 18; full triangles at $28.5 \mathrm{MeV}: \operatorname{R\in f} .19$. 
TABLE VII

Relative Differential Cross Sections for ${ }^{1} H(n, n)^{1} H$. Scale established by normalizing a Legendre fit to the total cross section.

\begin{tabular}{|c|c|c|c|c|c|}
\hline \multirow{2}{*}{ 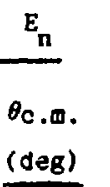 } & \multicolumn{2}{|c|}{$22.4 \mathrm{MeV}$} & \multicolumn{3}{|c|}{$23.7 \mathrm{MeV}$} \\
\hline & $\begin{array}{c}\sigma_{c . m} . \\
(\mathrm{mb} / \mathrm{sr})\end{array}$ & $\begin{array}{c}\text { error } \\
8\end{array}$ & $\begin{array}{l}\theta_{c . m} \\
\text { (deg) }\end{array}$ & $\underset{(m b / s r)}{\sigma_{c, m}}$ & $\begin{array}{c}\text { error } \\
\$\end{array}$ \\
\hline 28.38 & 33.2 & 4.3 & 53.13 & 31.0 & 2.4 \\
\hline 38.44 & 33.6 & 2.8 & 69.79 & 30.4 & 2.0 \\
\hline 49.09 & 35.6 & 4.0 & 99.25 & 31.1 & 1.5 \\
\hline 59.34 & 33.0 & 1.4 & & & \\
\hline 64.35 & 32.6 & 1.5 & & & \\
\hline 69.37 & 32.6 & 1.5 & & & \\
\hline 74.39 & 32.1 & 1.5 & & & \\
\hline 79.20 & 33.4 & 1.4 & & & \\
\hline 99.22 & 33.5 & 1.2 & & & \\
\hline 99.03 & 34.0 & 1.1 & & & \\
\hline 118.83 & 33.3 & 2.5 & & & \\
\hline
\end{tabular}

\begin{tabular}{|c|c|c|}
\hline $\begin{array}{l}\theta_{c . m .} \\
(d e g)\end{array}$ & $\begin{array}{c}\sigma_{n}, m . \\
(m b / s r)\end{array}$ & $\begin{array}{c}\text { error } \\
8\end{array}$ \\
\hline 40.29 & 26.7 & 8.0 \\
\hline 46.33 & 25.8 & 4.4 \\
\hline 50.35 & 24.8 & 4.4 \\
\hline 57.19 & 26.6 & 5.9 \\
\hline 60.40 & $27 \cdot 3$ & 4.3 \\
\hline 66.83 & 27.1 & 6.3 \\
\hline 70.44 & 26.5 & 4.0 \\
\hline 80.47 & 27.0 & 7.4 \\
\hline 90.49 & 25.8 & 4.1 \\
\hline $0 C .50$ & 26.0 & 4.2 \\
\hline 05.70 & 26.2 & 6.1 \\
\hline 10.50 & 28.4 & 4.8 \\
\hline
\end{tabular}

28.5 Med

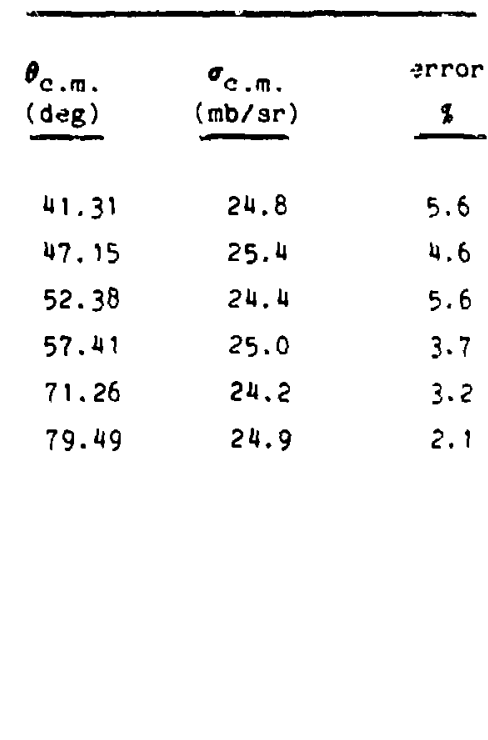




\section{REFERENCES}

1. M. Drosg, Nucl. Instrum. Meth. 105, 573 (1973).

2. J. D. Seagrave, J. C. Hopkins, D. R. Dixon, P. W. Keaton, Jr., E. C. Kerr, A. Nijier, R. H. Sherman, and R. K. Walter, Ann. Phys. (NY), 74, 250 (1972).

3. A. Niller, M. Drosg, J. C. Hopkins, J. D. Seagrave, and E. C. Kerr, Phys. Rev. C4, 36 (1971).

4. M. Drosg, D. K. McDaniels, J. C. Hopkins, J. D. Seagrave, R. H. Sherman, and E. C. Kerr, Phys. Rev. C9, 179 (1974).

5. M. Drosg, Nucl. Sci. Eng.s to be published.

6. H. Liskien and A. Paul sen, Nucl. Data Tables 11, 569 (1973).

7. C. A. Goulding, P. Stoler, and J. D. Seagrave, Nucl. Phys. B215, 253 (1973).

8. R. E. Shamu and J. G. Jenkin, Phys. Rev. 135, Bg9 (1964).

9. P. Hillman, R. H. Stahl, and N. F. Ramsay, Phys. Rev. 96, 115 (1954).

10. D. F. Measday and J. N. Palmieri, Nucl. Phys. 85, 129 (1966).

11. P. E. Tannenwald, Phys. Rev. 89,508 (1953).

12. LASL Physics and Cryogenics Groups, Nucl. Phys. 12, 291 (1959).

13. J. D. Seagrave, L. Cranberg, and J. E. Simmons, Phys. Rev. 119, 1981 (1960).

14. E. R. Flynn and P. J.Bendt, Phys. Rev. 128, 1268 (1962).

15. T. W. Burrows, Phys. Rev. C7, 1306 (1973).

16. L. N. Rothenberg, Phys. Rev. C1, 1226 (1970).

17. T. G. Masterson, Phys. Rev. C6, 690 (1972).

18. J. L. Fowler, M. Hussain, J. A. Cookson, C. A. Uttley, and R. B.

Schwartz, "Angular Distribution of Neutron-Proton Scattering at 27.3 MeV," Oak Ridge National Laboratory report ORNL-5306 (1977).

19. M. E. Remley, W. K. Jentschke, and P. G.Kruger, Phys. Rev. 89, 1194 (1953).

20. J. C. Hopkins and G. Breit, Nucl. Data Tables A9, 137 (1971).

21. M. Drosg, Proc. Int. Conf on the Interactions of Neutrons with Nuclei (E. Sheldon, ed.), Lowell, Mass., USA, p. 1383 (1976). 